\title{
Mg2+ and Mn2+ coordinate Cap-0-RNA to position substrates for efficient 2'-0- methyl transfer by SARS-CoV-2 nsp16
}

\author{
M. R. Lemus ${ }^{1}$, G. Minasov' ${ }^{2}$, L. Shuvalova ${ }^{3}$, N. L. Inniss' ${ }^{4}$, J. S. Brunzelle ${ }^{5}$, C. M. Daczkowski ${ }^{6}$, P. $^{2}$ \\ Hoover $^{7}$, A. D. Mesecar ${ }^{8}$, K. J. F. Satchell ${ }^{9}$ \\ ${ }^{1}$ Northwestern University ${ }^{2}$ CSGID, Department of Microbiology-Immunology, Northwestern \\ University, Feinberg School of Medicine, ${ }^{3}$ CSGID, Department of Microbiology-Immunology, \\ Northwestern University, Feinberg School of Medicine, ${ }^{4}$ CSGID, Department of Microbiology- \\ Immunology, Northwestern University, Feinberg School of Medicine, ${ }^{5}$ Northwestern Synchrotron \\ Research Center, Life Sciences Collaborative Access Team (LSCAT), Northwest, ${ }^{6}$ Department of \\ Biochemistry, Purdue University, ${ }^{7}$ Department of Microbiology-Immunology, Northwestern \\ University, Feinberg School of Medicine, ${ }^{8}$ Department of Biochemistry, Purdue University, ${ }^{9} \mathrm{CSGID}$, \\ Department of Microbiology-Immunology, Northwestern University, Feinberg School of Medicine \\ monica.lemus@northwestern.edu
}

Viral mRNAs must mimic the host mRNA to prevent degradation and activation of immune responses as well as promote translation. There are several mechanisms of capping depending of the virus, in coronaviruses, this process is mediated by non-structural proteins (nsp), encoded in the viral genome. The coronavirus SARS-CoV-2, the etiologic agent of the COVID-9 pandemic is a single strand positive RNA ( $\mathrm{ss}(+)$ RNA), which is capped at the 5' UTR. This process requires sequential reactions involving the transfer of a GMP moiety from GTP to the 5' end of the nascent $(+)$ ssRNA that is catalyzed by nsp13 and an unknown GTase. Then the N-7 methylation of the new attached guanidine, also named Cap-0 is catalyzed by the nsp14-nsp10 complex using S-adenosylmethionine as methyl donor (SAM) and followed by a 2'-O- methylation of the first ribonucleotide (Cap-1). The last reaction is catalyzed by the methyltransferase nsp16 in complex with activator nsp10. The reaction requires substrates: a short RNA and SAM, as well as Mg2+ or Mn2+, with preference for Mn2+. Here, we present the structures of nsp16nsp10 in complex with the substrates and products of the reaction determined by X-ray crystallography. These structures revealed a critical role of metal ions and as well as the length of the RNA in stabilizing interactions between ribonucleotides and nsp16. These interactions align the substrates to favor methyl transfer. Furthermore, we demonstrated that the reaction an aspartic residue that is highly conserved among coronaviruses alters the backbone conformation of the capped RNA in the active site. This aspartate is absent in mammalian methyltransferases and could be a promising site for designing coronavirus-specific inhibitors.

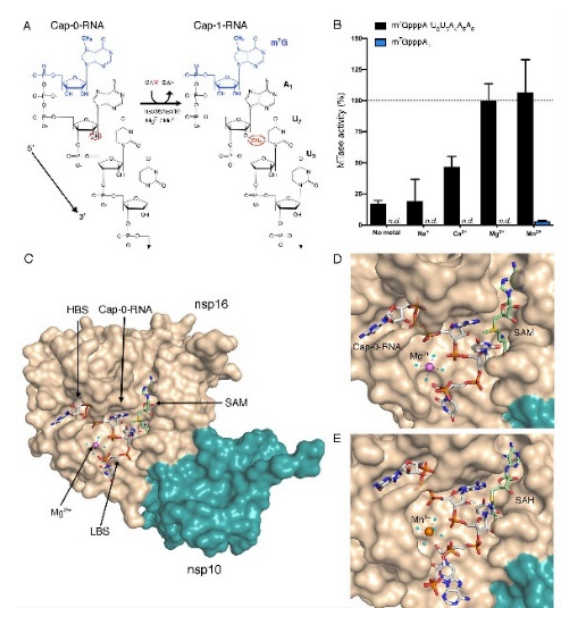

Figure 1 